\title{
Article \\ Two TGA Transcription Factor Members from Hyper-Susceptible Soybean Exhibiting Significant Basal Resistance to Soybean mosaic virus
}

\author{
Hua Jiang ${ }^{1,2,3,4,5}$, Shengyu Gu ${ }^{1,2,3,4,5}$, Kai Li ${ }^{1,2,3,4,5}$ and Junyi Gai $1,2,3,4,5, *$ \\ 1 Soybean Research Institute, Nanjing Agricultural University, Nanjing 210095, China; \\ jianghua_5220@126.com (H.J.); gsynau@163.com (S.G.); kail@njau.edu.cn (K.L.) \\ 2 MOA National Center for Soybean Improvement, Nanjing Agricultural University, Nanjing 210095, China \\ 3 MOA Key Laboratory of Biology and Genetic Improvement of Soybean (General), Nanjing Agricultural University, \\ Nanjing 210095, China \\ 4 State Key Laboratory for Crop Genetics and Germplasm Enhancement, Nanjing Agricultural University, \\ Nanjing 210095, China \\ 5 Jiangsu Collaborative Innovation Center for Modern Crop Production, Nanjing Agricultural University, \\ Nanjing 210095, China \\ * Correspondence: sri@njau.edu.cn; Tel.: +86-25-8439-5405
}

check for updates

Citation: Jiang, H.; Gu, S.; Li, K.; Gai, J. Two TGA Transcription Factor Members from Hyper-Susceptible Soybean Exhibiting Significant Basal Resistance to Soybean mosaic virus. Int. J. Mol. Sci. 2021, 22, 11329. https:// doi.org/10.3390/ijms222111329

Academic Editor: Hikmet Budak

Received: 22 September 2021

Accepted: 16 October 2021

Published: 20 October 2021

Publisher's Note: MDPI stays neutral with regard to jurisdictional claims in published maps and institutional affiliations.

Copyright: (c) 2021 by the authors. Licensee MDPI, Basel, Switzerland. This article is an open access article distributed under the terms and conditions of the Creative Commons Attribution (CC BY) license (https:/ / creativecommons.org/licenses/by/ $4.0 /)$.

\begin{abstract}
TGA transcription factors (TFs) exhibit basal resistance in Arabidopsis, but susceptibility to a pathogen attack in tomatoes; however, their roles in soybean (Glycine max) to Soybean mosaic virus (SMV) are unknown. In this study, 27 TGA genes were isolated from a SMV hyper-susceptible soybean NN1138-2, designated GmTGA1 GmTGA27, which were clustered into seven phylogenetic groups. The expression profiles of GmTGAs showed that the highly expressed genes were mainly in Groups I, II, and VII under non-induction conditions, while out of the 27 GmTGAs, 19 responded to SMV-induction. Interestingly, in further transient $N$. benthamiana-SMV pathosystem assay, all the 19 GmTGAs overexpressed did not promote SMV infection in inoculated leaves, but they exhibited basal resistance except one without function. Among the 18 functional ones, GmTGA8 and GmTGA19, with similar motif distribution, nuclear localization sequence and interaction proteins, showed a rapid response to SMV infection and performed better than the others in inhibiting SMV multiplication. This finding suggested that GmTGA TFs may support basal resistance to SMV even from a hypersusceptible source. What the mechanism of the genes (GmTGA8, GmTGA19, etc.) with basal resistance to SMV is and what their potential for the future improvement of resistance to SMV in soybeans is, are to be explored.
\end{abstract}

Keywords: soybean (Glycine max (L.) Merr.); TGA transcription factor; molecular characterization; Soybean mosaic virus; Nicotiana benthamiana; basal resistance

\section{Introduction}

In general, plants show a resistance or susceptibility response in facing a pathogen attack, which usually involves dramatic changes of gene expression across multiple signaling and metabolism pathways [1,2]. Thereinto, a large number of genes that encode regulatory proteins were expropriated, among which transcription factors (TFs) are typical examples [3]. During plant growth and development, in defense responses and disease development, TFs often serve as transcriptional regulators in the nucleus and play a key role in manipulating gene expression by binding to specific cis-regulatory elements in the promoters of target genes [4].

TGA TF belong to Group D of the basic leucine zipper gene family, which is the largest TF family in plants [5]. This type of TF was first identified by their ability in binding to the activating sequence 1 element of the Cauliflower mosaic virus 35S promoter [6]. Because the activating sequence 1 was typically composed of a tandem of the sequence TGACG 
with approximately 21 bases in length, the TGACG-sequence-specific binding proteins were named as TGA proteins [7]. Initially, the sequence analysis of two TGA cDNA clones isolated from tobacco showed both proteins containing a basic leucine zipper (bZIP) domain composed of both basic and zipper regions [6]. The basic region usually contained the nuclear localization signal (NLS), which was rich in arginine and lysine, interacting with the major groove of the DNA double helix. While the zipper region was an amphipathic helix of 30-40 residues with every seventh residue a leucine, which was required to hold together two DNA binding regions [5]. The bZIP proteins commonly formed homodimers or heterodimers to perform their function, and specificity was determined by the non-leucine residues in the zipper region [5]. Since the first TGA1a gene was found in tobacco [6], many TGA TFs have been identified in plants, such as in the dicotyledonous plant Arabidopsis, there were ten members [8]. Similarly, four TGA TFs and 11 TGA-like proteins were also identified in the monocotyledonous plant rice [9].

After the advent of Arabidopsis as one of the prime model systems for plant molecular biology, the TGA family analysis was mainly performed in this species [10]. The TGA family was grouped into five clades according to the amino acid sequence similarity in Arabidopsis $[5,10]$. Among them, TGA1 and TGA4 belonged to clade I, TGA2, TGA5, and TGA6 belonged to clade II, TGA3 and TGA7 belonged to clade III, TGA9 and TGA10 belonged to clade IV, and PERIANTHIA/TGA8 belonged to clade V [10]. The clade II TGA TFs were identified as interaction partners of the ankyrin repeat protein NPR1 [NONEXPRESSOR OF pathogenesis-related (PR) GENES 1] to activate NPR1 target genes expression through directly binding to their cis-elements [11]. NPR1 was a key regulator of $P R$ genes expression and was required to establish the systemic acquired resistance (SAR) [12]. The knockout analysis of Arabidopsis TGA2, TGA5, and TGA6 revealed their redundant and positive roles in SAR, whereas the TGA triple knockout plants were not impaired in basal resistance to the bacterial pathogen Pseudomonas syringaepv maculicola ES4326 [12]. Though the clade I TGA TFs did not interact with NPR1 in the yeast two-hybrid system, they could interact in SA-treated leaves, and this suggested that TGA acted independently of NPR1 or on the upstream of SA biosynthesis and NPR1-dependent signaling to positively regulate basal resistance [13-16]. In contrast, TGA1.a-TF promoted disease development caused by Botrytis cinerea in tomato by suppressing the expression of two jasmonic aciddependent defense genes, proteinase inhibitors I and II [17]. Furthermore, clade III TGA3 played a positive role in basal resistance, which recruited cytokinin-activated TF ARR2 to SA-responsive defense promoters through direct protein-protein interaction, while TGA7 does not $[18,19]$. In addition, clade IV TGA9 and TGA10 were redundantly required for anther development and were involved in ROS-mediated responses to bacterial PAMP flg22 [20,21]. Similarly, PERIANTHIA/TGA8 TF also played a role in flower development, which was a repressor of petal formation [22]. These findings indicated that TGA proteins played critical roles in pathogen-induced basal resistance, susceptibility and SAR (Figure S1), which suggested the homologous genes of Arabidopsis TGA TFs in different plant species may also be functional diversification.

Soybean (Glycine max (L.) Merr.) is an economically important oil and protein crop. Soybean mosaic virus (SMV) is a prevalent viral pathogen of soybean [Glycine max (L.) Merr.], found especially in China [23-25], usually leads to soybean yield reductions ranging from $8 \%$ to $100 \%$ [26]. SMV belongs to Potyvirus, its genome is a single-stranded, positive-sense (+) RNA, with a length of $\sim 9.6 \mathrm{~Kb}$. In general, SMV's host range is restricted mostly to cultivated soybean and wild soybean [27]. Thereinto, the cultivated soybean NN1138-2 is hyper-susceptible to 22 SMV strains identified in China [24]. Besides, N. benthamiana plants, as an ideal model plant for the investigation of plant-pathogen interaction was also systemically infected by some SMV isolates [28-30], which are helpful for studying the function of soybean endogenous genes to SMV stress.

To date, most TGA TFs studies have been carried out in model plant Arabidopsis, but there is a lack of reports on the potential functions (susceptibility, or resistance, or neutral) of soybean TGA TFs under SMV stress. In this study, we intend to isolate GmTGA TFs 
from an SMV hyper-susceptible soybean cultivar NN1138-2, classify them into different phylogenetic groups, detect their expression levels under non-inducing and SMV-inducing condition, and evaluate the function of GmTGAs to SMV challenge to understand the potential of GmTGA TFs for improving the self-defense of susceptible host soybean or promoting SMV to successfully colonize the host.

\section{Results}

\subsection{Identification and Phylogenetic Groups of GmTGAs in Soybeans}

We are interested in TGA TFs because the literature reported that these kinds of TFs were involved in the disease processes $[16,17,19]$. The characteristic analysis of gene families in soybean is often based on the sequences of the nucleic acid and amino acid derived from the reference genome of Williams 82 [31]. Differently, we aim to uncover the characteristics of GmTGA gene family in the SMV-susceptible soybean cultivar NN1138-2. Firstly, BLASTP and HMM searches were performed against the reference protein of soybean Williams 82 to identify GmTGA. As a result, 25 and 27 GmTGA genes were searched respectively. Twenty five out of 27 GmTGA genes were consistent and the remaining two genes were GmTGA18 and GmTGA21 (Table 1). Additionally, all identified TGA TFs contained classical bZIP and DOG1 domains based on SMART and Pfam analysis. Based on this, the coding sequences of 27 TGA TFs in cultivar NN1138-2 were obtained by homology cloning, and the primers used were listed in Table S1. As shown in Table 1, all GmTGAs were named GmTGA1 27, depending on their location on the chromosomes and Gene ID number in soybean. These genes were widely distributed on 16 out of 20 chromosomes with a maximum of three TGA genes on chromosome 3, 10 and 13, respectively. Meanwhile, variations of the open reading frame, amino acid residues, molecular weight, and protein isoelectric points were predicted, it suggested the presence of putative novel variants in the gene family (Table 1).

Table 1. Characteristics and nomenclature of soybean TGA transcription factors in soybean cultivar NN1138-2.

\begin{tabular}{|c|c|c|c|c|c|c|}
\hline Gene Name & $\begin{array}{l}\text { Gene Model Name } \\
\text { (Wm82.a2.v1) }\end{array}$ & Location Coordinates & ORF (bp) & Size (aa) & MW (kDa) & $\mathrm{p} I$ \\
\hline GmTGA1 & Glyma.01G084200 & Chr01:24682405..24689210(-) & 1461 & 486 & 54.31 & 7.32 \\
\hline GmTGA2 & Glyma.02G097900 & Chr02:8834516..8841154(-) & 1404 & 467 & 51.85 & 7.32 \\
\hline GmTGA3 & Glyma.02G176800 & Chr02:29019977..29028838 (-) & 1455 & 484 & 53.93 & 7.77 \\
\hline GmTGA4 & Glyma.03G127600 & Chr03:34171878..34178336 (-) & 1383 & 460 & 50.93 & 7.00 \\
\hline GmTGA5 & Glyma.03G128200 & Chr03:34259590..34264026 (+) & 873 & 290 & 32.37 & 8.82 \\
\hline GmTGA6 & Glyma.03G142400 & Chr03:35810204..35817330 (-) & 1476 & 491 & 55.20 & 8.22 \\
\hline GmTGA7 & Glyma.04G254800 & Chr04:52132354..52137159(-) & 1089 & 362 & 40.93 & 8.69 \\
\hline GmTGA8 & Glyma.05G182500 & Chr05:37029299..37037313 (+) & 1113 & 370 & 42.08 & 7.11 \\
\hline GmTGA9 & Glyma.06G107300 & Chr06:8630095..8635111 (+) & 1068 & 355 & 39.98 & 6.21 \\
\hline GmTGA10 & Glyma.08G140100 & Chr08:10719899..10725773 (+) & 1140 & 379 & 43.11 & 7.78 \\
\hline GmTGA11 & Glyma.10G092100 & Chr10:12738719..12747206 (+) & 1554 & 517 & 57.92 & 6.61 \\
\hline GmTGA12 & Glyma.10G276100 & Chr10:49858890..49863059 (-) & 1371 & 456 & 50.99 & 6.08 \\
\hline GmTGA13 & Glyma.10G296200 & Chr10:51306677..51314923 (-) & 999 & 332 & 37.14 & 8.94 \\
\hline GmTGA14 & Glyma.11G183700 & Chr11:25149986..25158384 (+) & 1476 & 491 & 54.01 & 6.04 \\
\hline GmTGA15 & Glyma.11G236300 & Chr11:33112560..33118319(-) & 1095 & 364 & 41.18 & 6.28 \\
\hline GmTGA16 & Glyma.12G088700 & Chr12:7269137..7277440(-) & 1506 & 501 & 55.25 & 6.61 \\
\hline GmTGA17 & Glyma.12G184500 & Chr12:34579255..34588903 (-) & 1467 & 488 & 54.12 & 6.75 \\
\hline GmTGA18 & Glyma.13G085100 & Chr13:19659235..19664662 (-) & 1113 & 370 & 41.92 & 7.13 \\
\hline GmTGA19 & Glyma.13G193700 & Chr13:30682181..30695761 (-) & 1410 & 469 & 51.99 & 5.98 \\
\hline GmTGA20 & Glyma.13G316900 & Chr13:41139769..41147330 (+) & 1473 & 490 & 54.63 & 6.96 \\
\hline GmTGA21 & Glyma.14G167000 & Chr14:41307404..41312938 (-) & 1113 & 370 & 41.87 & 8.26 \\
\hline GmTGA22 & Glyma.15G232000 & Chr15:43630502..43639894 (-) & 1344 & 447 & 49.45 & 5.86 \\
\hline GmTGA23 & Glyma.18G020900 & Chr18:1529888..1535807 (+) & 1089 & 362 & 41.05 & 7.78 \\
\hline GmTGA24 & Glyma.19G130200 & Chr19:38989153..38995505(-) & 1380 & 459 & 50.72 & 8.53 \\
\hline GmTGA25 & Glyma.19G145300 & Chr19:40604030..40611501 (-) & 1473 & 490 & 55.20 & 8.51 \\
\hline GmTGA26 & Glyma.20G113600 & Chr20:35557820..35562522(+) & 1368 & 455 & 50.70 & 5.88 \\
\hline GmTGA27 & Glyma.20G246400 & Chr20:47647701..47654066 (-) & 1335 & 444 & 49.51 & 5.91 \\
\hline
\end{tabular}


According to the phylogenetic tree of all 27 GmTGAs from NN1138-2, seven groups (I to VII) were clustered (Figure 1A), which was similar to the previous report on subclassification within TGA family proteins [32]. GmTGA13, 19, 22 and 27 were assigned to Group I; GmTGA4, 5 and 24 were assigned to Group II; GmTGA1, 2, 12 and 26 were assigned to Group III; GmTGA3, 6, 11, and 25 were assigned to Group IV; GmTGA14, 16, 17 and 20 were assigned to Group V; GmTGA7, 9, 18 and 21 were assigned to Group VI; GmTGA8, 10, 15 and 23 were assigned to Group VII. Among them, only Group II contained three GmTGAs and others contained four GmTGAs. Besides, phylogenetic analyses showed a close relationship between soybean TGA members and Arabidopsis TGA family (Figure S2), while the addition of Arabidopsis TGA proteins did not affect the grouping of the phylogenetic tree (Figure S2). As expected, most of the GmTGA genes clustered in pairs, except GmTGA5, and Group I was the largest with seven members of soybean and Arabidopsis (Figure S2). In addition, a distinct phylogenetic branch, annotated as GmTGA4, 5 and 24, was not easily linked to any of the AtTGA orthologues, suggested that this group of TGA members were specific to the soybean genome.

$\mathbf{A}$

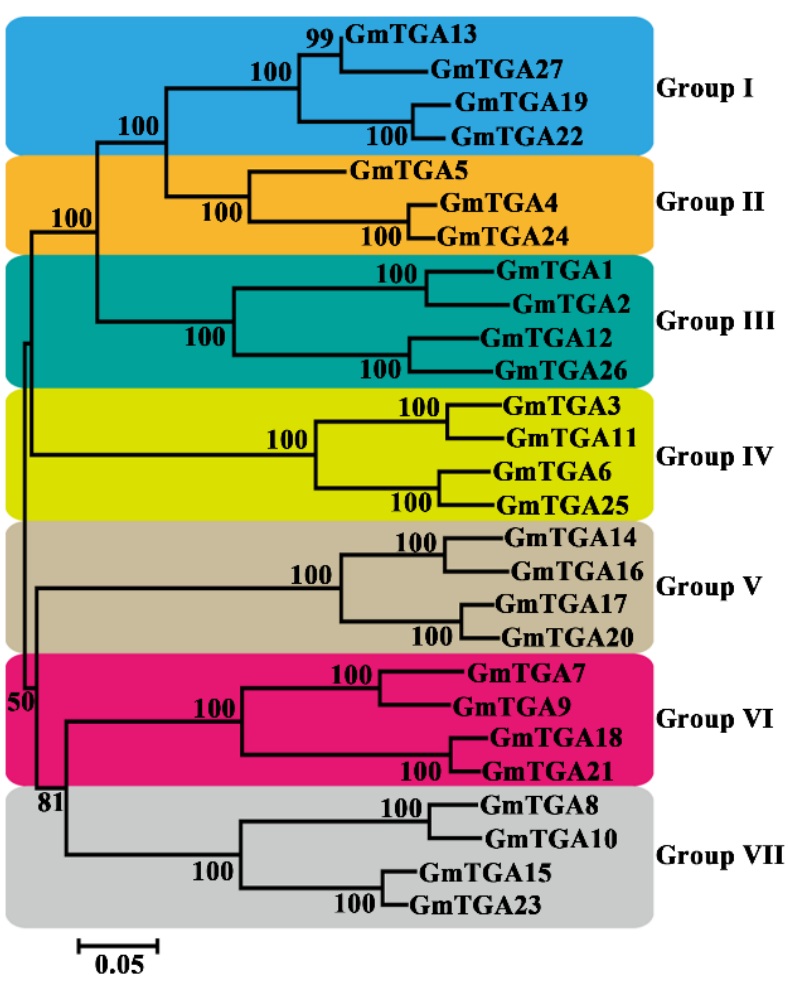

B

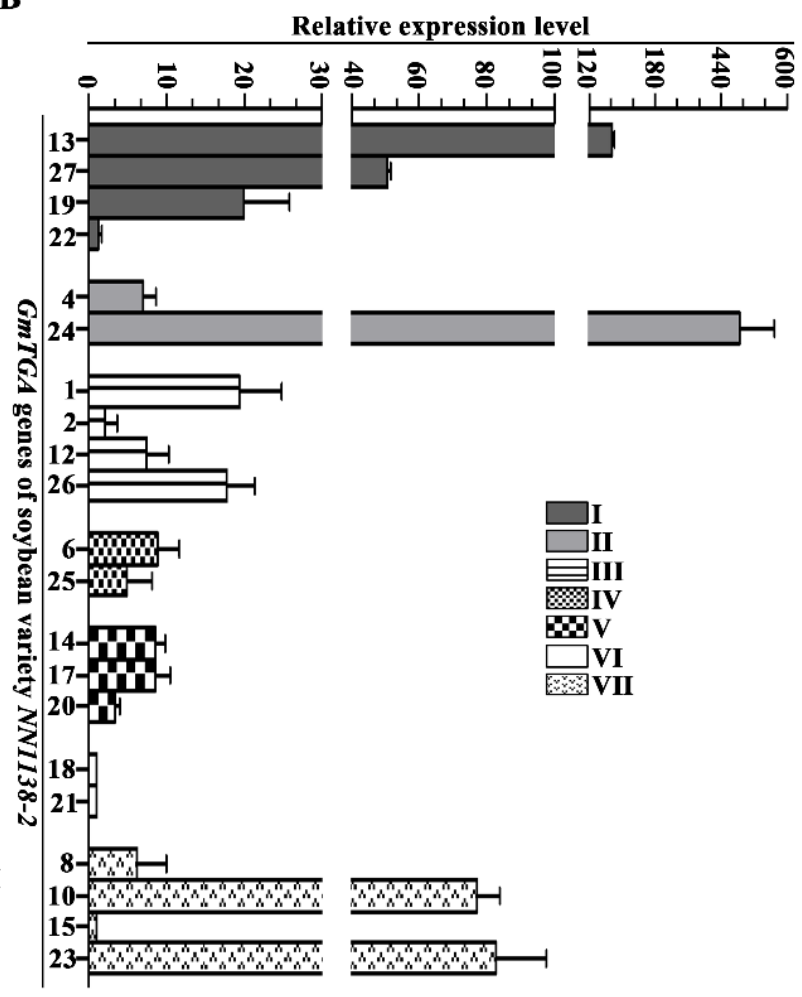

Figure 1. Phylogenetic relationship analysis of 27 GmTGA proteins of soybean NN1138-2 and expression profile of these GmTGA genes in the unifoliate leaves. (A) Phylogenetic relationship analysis of 27 GmTGA proteins. The amino acid sequences of 27 GmTGA proteins from soybean NN1138-2 were used to construct the neighbor-joining (NJ) tree using MEGA 6.0 with 1000 bootstrap replicates, from which 7 groups of GmTGA were identified. (B) Expression profile of GmTGA genes in fully developed unifoliate leaves tissue. The mRNA transcript levels of 27 GmTGA genes were analyzed using qRT-PCR in the unifoliate leaves. Out of them, the fluorescence signal of 21 GmTGA genes were detected while others not. GmEF1B and GmActin11 were used as internal controls. All experiments were performed with three independent biological repeats. The error bars represent the standard deviation.

\subsection{Differential Expression Profiles of GmTGAs in the Unifoliate Leaves of Soybean}

To explore expression patterns of seven groups of GmTGA genes in soybean plants at

VC stage, qRT-PCR was performed using gene-specific primers. The relative expression level of 21 out of 27 GmTGA genes was shown in Figure 1B. Similar to the transcriptome 
data of soybean Williams 82 in the phytozome database, the fluorescence signal of the GmTGA3, 5, 7, 9, 11, and 16 belonging to four different groups (II, IV, V and VI) were not detected. It indicated that these six genes were not expressed in the unifoliate leaves during the VC development stage of soybean. As shown in Figure 1B, there were differences in the relative expression level of seven groups of GmTGA genes. Among them, the genes in Groups I, II and VII were very highly expressed, but in Groups III, IV, V and VI they were not, especially in Group VI where they were almost not expressed. Moreover, the relative expression level also varied in the same group (Figure 1B). Additionally, out of 21 GmTGA genes, eight genes expressed relatively higher, that is, GmTGA1, 10, 13, 19, 23, 24, 26 and 27, five genes expressed relatively lower, that is, GmTGA2, 15, 18, 21 and 22, and others were between them.

\subsection{Differential Responses of GmTGA Genes to SMV Challenge}

In general, the unifoliate leaves were used for artificial SMV-inoculation in soybean. To investigate the potential roles of GmTGA genes in response to SMV challenge; the expression profiles of GmTGA genes in unifoliate leaves were examined at 1, 2, 4, 8, 12, 24 and $72 \mathrm{~h}$ post-inoculation (hpi) using qRT-PCR. The relative expression levels of 19 out of 21 GmTGA genes under Mock and SMV treatment were shown in Figure 2 and Figure S3. The cycle threshold value of both GmTGA18 and 21 were consistent (equal to 35 ) at different time points before and after SMV inoculation, indicating Group VI was not involve in the SMV disease process. The transcript levels of GmTGA genes showed differences among and within the six TGA groups (I to V, and VII), which suggested the different transcriptional response of these GmTGA genes to SMV inoculation. At $1 \mathrm{hpi}$, most of the GmTGA genes were down-regulated and only GmTGA2 and GmTGA15 were slightly up-regulated. GmTGA4, 8, 10, 13 and 19 showed a rapid response to SMV inoculation at earlier $2 \mathrm{~h}$ time points; while GmTGA12 and 26 showed an intense response at $24 \mathrm{hpi}$ (Figure 2). In contrast, GmTGA14 was down-regulated throughout each inoculation time point (Figure S3). Among them, GmTGA13 and 19 belong to Group I, GmTGA4 belong to Group II, GmTGA12 and 26 belong to Group III, GmTGA14 belong to Group V, and GmTGA8 and 10 belong to Group VII, which indicated that Groups I, II, III, V and VII may be mostly involved in response to SMV-stress. Besides, GmTGA1, 6, 17, 20, 22, 24, 25 and 27 were also induced. In short, our results suggest that a part of the GmTGA genes may be required for the host soybean self-defense or SMV successful colonization in soybean.

\subsection{Transient Overexpression of GmTGA Genes in Nicotiana benthamiana Inhibiting but Not Promoting SMV-Multiplication}

In a previous study, we found that $N$. benthamiana plants can be systemically infected by an SMV isolate 4278-1 [28]. The N. benthamiana-SMV-4278-1 pathosystem combined with Agrobacterium-mediated transient expression assay was a potential for exploring the function of GmTGA genes to SMV. Focusing on the putative importance of the GmTGA genes' response to SMV-induction on improving or inhibiting SMV multiplication, the leaves of $\sim 4$ weeks $N$. benthamiana seedlings were infiltrated by Agrobacterium tumefaciens that contain each overexpression construct of the 19 GmTGA genes responded to SMVinduction, followed by the mechanical inoculation of the SMV isolate 4278-1, and the viral contents were further detected by ELISA assay at $3 \mathrm{dpi}$. The results showed 18 out of 19 GmTGA genes were positive for inhibiting SMV multiplication, except GmTGA 6 with no effect on SMV multiplication compared to empty vector control (Table 2). Moreover, the effects of most overexpressed GmTGA genes were similar to positive control (SMV-cp gene overexpression) on inhibiting SMV multiplication for SMV-inoculated leaves in three independent experiments (Table 2). Among them, GmTGA8 and GmTGA19 had a superior performance to positive control and other GmTGA genes. The two genes, belonging to Groups I and VII, respectively, responded promptly to SMV infection and inhibited strongly to SMV multiplication (Figure 2 and Table 2). It is unexpected that no one promoted SMV multiplication in inoculated leaves, which indicated the GmTGA genes were not responsible for the susceptibility to SMV in hyper-susceptible soybean but benefiting for the basal 
resistance to SMV (Table 2). It also suggested that GmTGA genes may be functionally redundant for inhibiting SMV multiplication. Taken together, multiple GmTGA genes were recruited by the SMV-susceptible host soybean under SMV challenge and served a positive role in self-defense. Especially, the genes with rapid-response to SMV-infection, GmTGA8 and 19, may have certain potential for the improvement of SMV-resistance for SMV-susceptible soybeans.
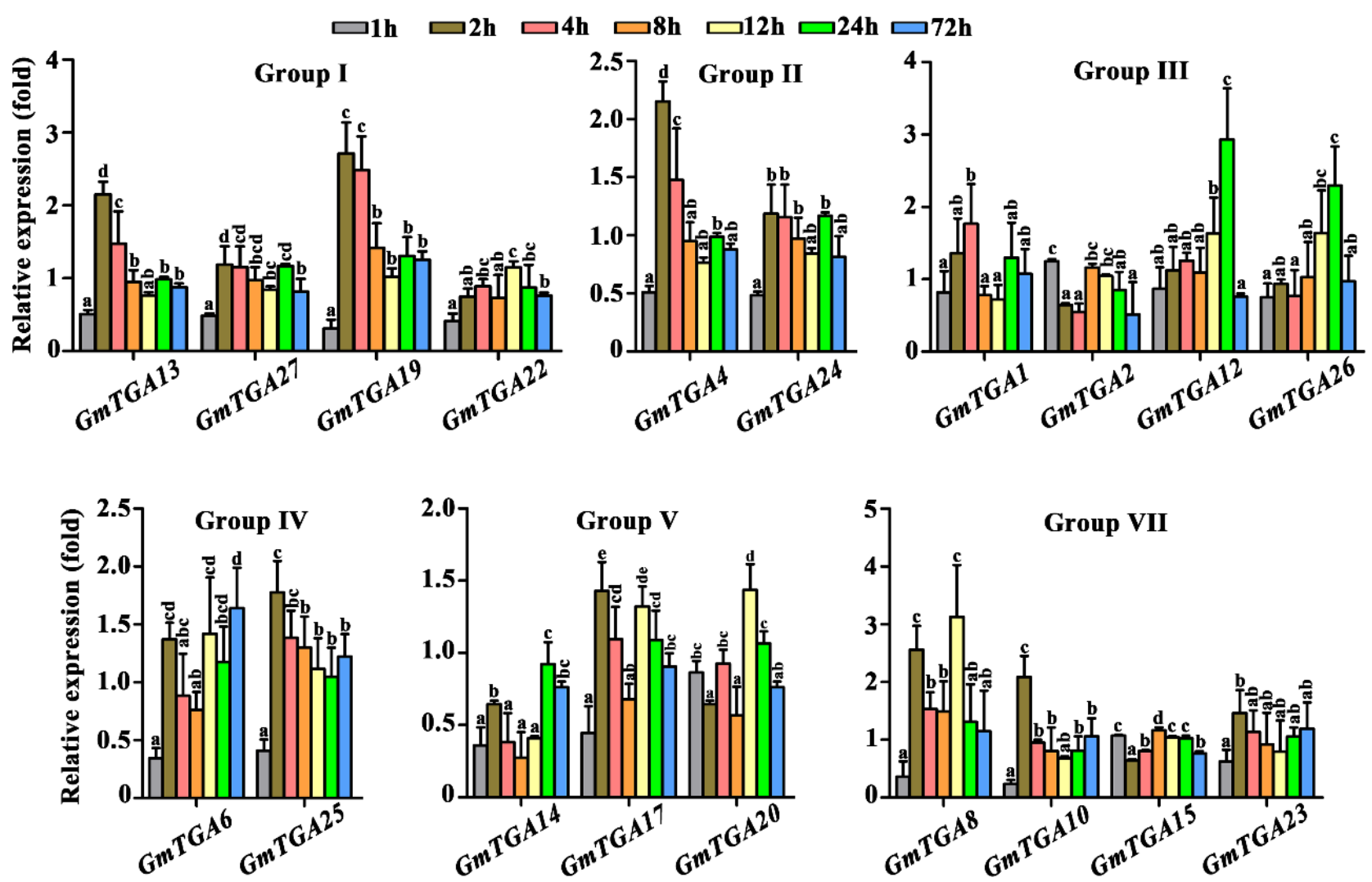

Figure 2. Expression profiles of GmTGA genes of soybean NN1138-2 following infection with SMV at 1, 2, 4, 8, 12, 24 and $48 \mathrm{~h}$ post-inoculation (hpi). RNAs isolated from non-inoculated and inoculated unifoliate leaves of soybean were employed to analyze the expression of 7 groups of GmTGA genes using qRT-PCR. The relative expression levels were calculated by comparing the gene-expression values in inoculated vs. non-inoculated unifoliate leave tissues using the $2^{-\Delta \Delta \mathrm{Ct}}$ method. The data are presented as mean \pm standard deviation from three independent experiments. Relative quantitative values in the same gene with different letters are significantly different at $p=0.05$ level. 
Table 2. The virus content analysis in N. benthamiana leaves overexpressed with different GmTGA genes under SMV inoculation by DAS-ELISA at 3dpi.

\begin{tabular}{|c|c|c|c|c|c|c|c|c|c|c|c|c|c|c|c|c|c|c|c|c|c|c|}
\hline \multicolumn{3}{|c|}{ Sample Name } & \multicolumn{19}{|c|}{ SMV-Induced GmTGA Transcription Factors } & \multirow{3}{*}{+} \\
\hline & & \multirow[t]{2}{*}{ EV } & \multicolumn{4}{|c|}{ Group I } & \multicolumn{2}{|c|}{ Group II } & \multicolumn{4}{|c|}{ Group III } & \multicolumn{2}{|c|}{ Group IV } & \multicolumn{3}{|c|}{ Group V } & \multicolumn{4}{|c|}{ Group VII } & \\
\hline Treatment & & & $\# 13$ & $\# 27$ & $\# 19$ & $\# 22$ & $\# 4$ & $\# 24$ & $\# 1$ & $\# 2$ & $\# 12$ & $\# 26$ & $\# 6$ & $\# 25$ & $\# 14$ & $\# 17$ & $\# 20$ & $\# 8$ & $\# 10$ & $\# 15$ & $\# 23$ & \\
\hline Mock & & 0.10 & 0.11 & 0.13 & 0.12 & 0.11 & 0.10 & $0.12 \mathrm{c}$ & $0.12 \mathrm{c}$ & 0.12 & $0.12 \mathrm{c}$ & $0.12 \mathrm{c}$ & 0.11 & $0.12 \mathrm{c}$ & 0.11 & 0.12 & 0.12 & 0.12 & 0.12 & 0.11 & 0.13 & 0.12 \\
\hline SMV & E1 & $\begin{array}{l}0.74^{\mathrm{a}} \\
0.81^{\mathrm{c}}\end{array}$ & $0.41^{\mathrm{oc}} \mathrm{b}$ & $0.40^{\mathrm{oc}} \mathrm{b}$ & $0.28^{\mathrm{a}} \mathrm{a}$ & $\begin{array}{l}0.43^{\mathrm{DC}} \\
0.5\end{array}$ & 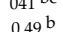 & $0.45^{2} \mathrm{~b}$ & $0.47^{\mathrm{b}}$ & $0.41^{\mathrm{DC}}$ & $\begin{array}{l}0.47^{\mathrm{c}} \\
0.5 \mathrm{~b}\end{array}$ & $0.5^{\mathrm{c}} \mathrm{b}$ & $\begin{array}{l}0.74^{\mathrm{d}} \\
0.8 \mathrm{c}\end{array}$ & $0.45^{\mathrm{c}} \mathrm{b}$ & $0.41^{\mathrm{bc}}$ & $\begin{array}{l}0.47^{\mathrm{c}} \\
0.5 \mathrm{~b}\end{array}$ & $0.46^{\mathrm{c}}$ & $0.30^{\mathrm{a}}$ & $0.42 \mathrm{bc}$ & $034 \mathrm{ab}$ & $0.51^{\mathrm{c}}$ & $0.40^{\mathrm{bc}}$ \\
\hline & E3 & $0.72^{\mathrm{c}}$ & $0.42 \mathrm{~b}$ & $0.45^{0} \mathrm{~b}$ & $0.28^{\mathrm{a}}$ & $0.41^{\mathrm{b}}$ & $0.43^{\circ} \mathrm{b}$ & $0.40^{\mathrm{b}}$ & $0.40^{\mathrm{b}}$ & $0.39 \mathrm{~b}$ & $0.40^{\mathrm{b}}$ & $0.036 \mathrm{~b}$ & $0.70^{\circ} \mathrm{c}$ & $0.034^{\circ}$ & $0.44^{\circ} \mathrm{b}$ & $0.045^{\circ} \mathrm{b}$ & $0.036 \mathrm{~b}$ & $0.27 \mathrm{a}$ & $0.33^{6}$ & $0.46 \mathrm{~b}$ & $0.58^{\circ} \mathrm{b}$ & $\begin{array}{r}0.47^{\circ} \\
0.42^{b}\end{array}$ \\
\hline
\end{tabular}

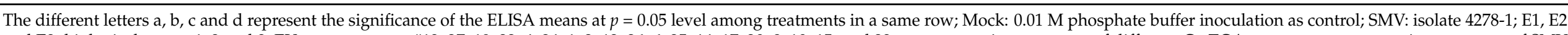

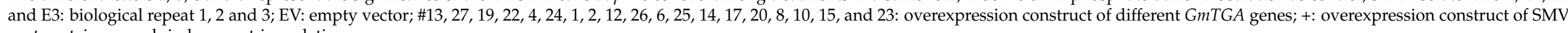
coat protein gene; dpi: days post-inoculation. 


\subsection{Characteristics and Interaction Network of GmTGA 8 and GmTGA19 Proteins}

In view of soybean genes, GmTGA8 and GmTGA19 were superior to others in inhibiting SMV-multiplication; their conserved motifs were predicted based on the MEME online tool, using "searching for ten motifs" and leaving the rest setting kept as default. The results showed that GmTGA8 and GmTGA19 contained four conserved motifs with a significance level of 0.05. As shown in Figure 3A, the width of motif 1 to motif 3 was 50, while for motif 4 it was 26 . Among them, motif 2 showed the highest identity of $82 \%$, and was rich in arginine $(\mathrm{R})$ as well as glutamine $(\mathrm{Q})$. Besides, we found that the two TGA proteins exhibited the same motifs in C-terminal regions (motifs 1 to 4 ), suggested the Cterminal part of TGA proteins was conserved, whereas the $\mathrm{N}$-terminal region was divergent (Figure 3). Due to the TF acting in the nucleus, the NLS of GmTGA8 and GmTGA19 was predicted by the NLStradamus online tool [33], and the same nuclear localization sequence "REAARKSRLRKK" located in the motif 2 region was discovered among them.

$\mathbf{A}$

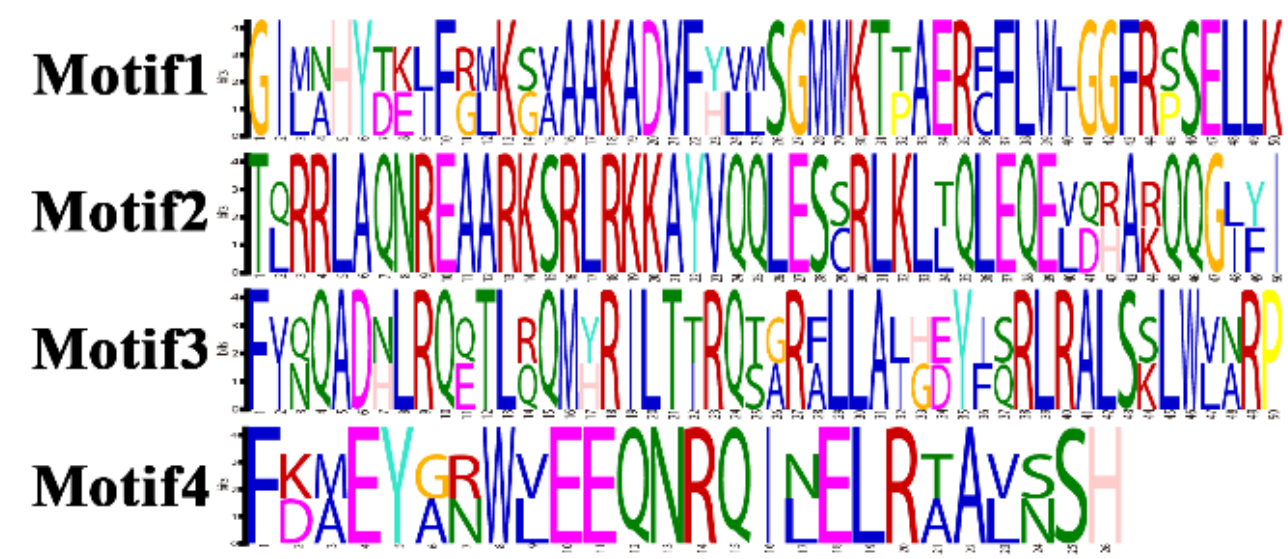

B

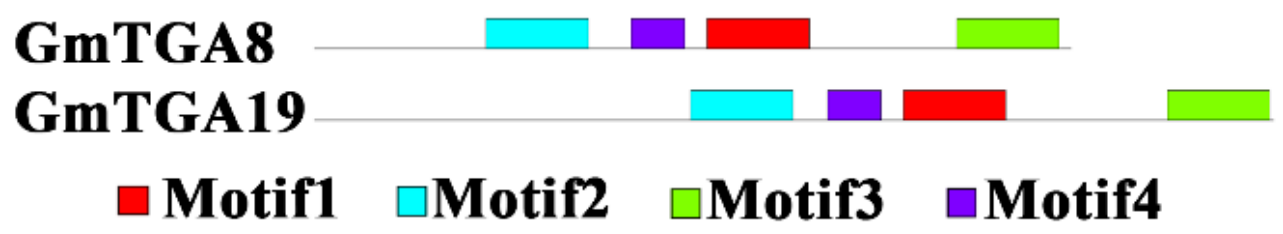

Figure 3. Conserved motifs in GmTGA 8 and 19 protein using MEME-suite. (A) The conserved motifs between GmTGA 8 and 19 proteins. The second motif covers the nuclear localization signals (NLS). Only the motifs with $E$-values $<0.05$ as well as no overlap with each other were displayed. (B) The distribution of motifs along with the protein sequences. Gray lines represent the nonconserved sequences, and each motif is represented by a box numbered at the bottom.

To identify the interaction proteins of GmTGA8 and GmTGA19 in soybean, the two TGA protein sequences as input were used for constructing the PPI networks through the STRING online tool (Figure 4). As shown in Figure 4A, the GmTGA8 protein showed interactions with 11 proteins, which were divided into six categories, including NPR1, NPR1-related, NPR3-related, cysteine-rich secretory protein-related, leghemoglobin-related and V5/TPX-1 related proteins. Similarly, the GmTGA19 protein showed interactions with ten proteins, which were divided into five categories, including NPR1, NPR1-related, NPR3-related, EDS1 and WRKY8/41 proteins (Figure 4B). As expected, the similar types of proteins were retrieved in both PPI networks, such as the NPR class proteins, in which NPR1 was a key regulator of the SA-mediated SAR in Arabidopsis [34]. In addition, we also found specific interaction proteins in both PPI networks, indicating that the regulatory network 
between GmTGA8 and GmTGA19 proteins were distinct. Thus, identifying the potential associated genes of GmTGA8 and GmTGA19 was helpful for understanding and exploring their possible regulatory mechanisms in soybean when suffering from SMV challenge.

A

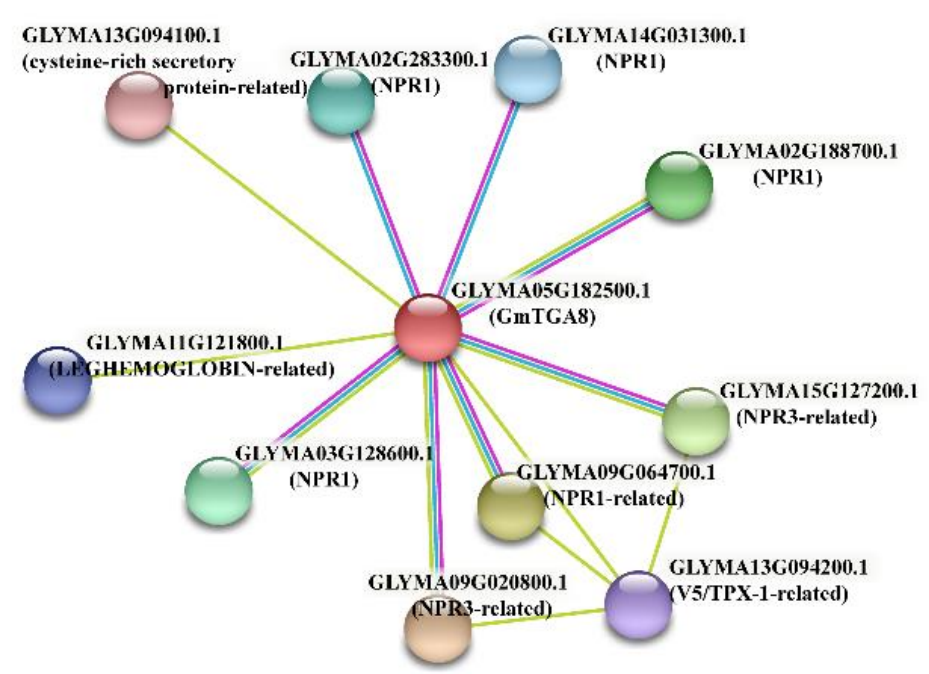

B

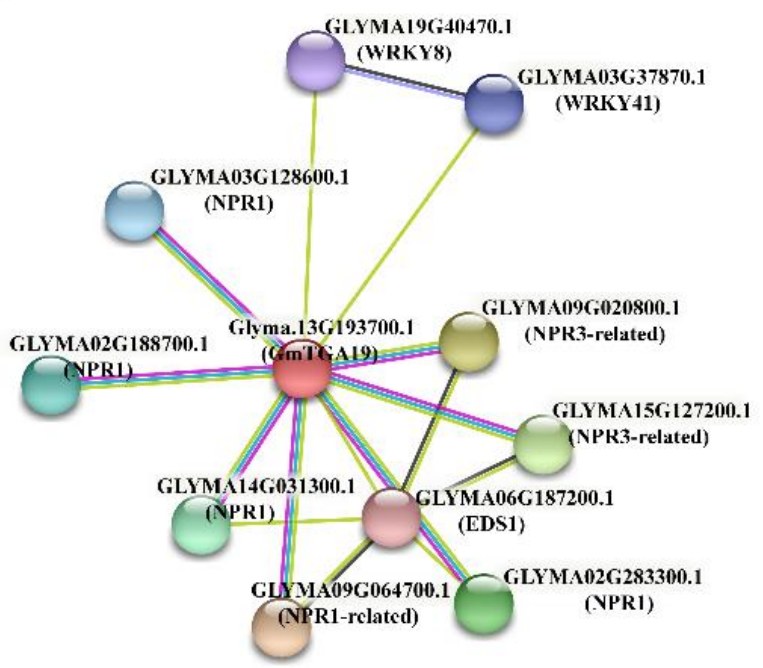

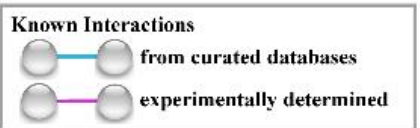

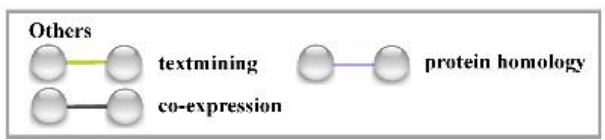

Figure 4. STRING interaction diagram of the analyzed GmTGA 8 (A) and 19 (B) in soybean. Each filled node denotes a gene and protein product of each gene is predicted based on phytozome online database; edges between nodes indicate interactions between protein products. Different edge colors represent the existence of different types of evidence used in predicting the associations. The first shell was set to no more than ten interactors and no interactor was set in second shell.

\section{Discussion}

\subsection{Characteristics of the GmTGA Family in Response to SMV Stress}

In this study, a total of 27 GmTGA genes isolated from a SMV hyper-susceptible soybean NN1138-2 were identified and analyzed for their characteristics and expression levels under non-inducing and SMV-inducing conditions. Obviously, the TGA gene number in soybean was more than that in other plant species, which might come from the expansion of the soybean gene family caused by soybean-specific segmental duplications $[6,8,9,31]$. In general, the large number of TGA genes could be interpreted as a possible functional redundancy and/or sub- or neo-functionalization of this protein family in soybean.

The expression patterns of GmTGA genes under different stress stimuli might provide insights into the functional divergence in soybean. The expression of the GmTGA genes in soybean NN1138-2 varied at the soybean VC development stage, which indicated that some GmTGA proteins may be recruited for tissue development (Figure 1). Arabidopsis TGA proteins often played a vital role in growth development, such as AtTGA9 and AtTGA10, which were expressed throughout early anther primordia and redundantly required for another development [20]. However, unlike Arabidopsis, there have been barely any reports regarding GmTGA genes regulating plant development in soybean.

Additionally, TGA TFs responded differentially to SMV-infection, some GmTGA genes ( $G m T G A 4,8,10,13$, and 19) showed a rapid response, while some (GmTGA12 and 26) were slower (Figure 2 and Figure S3). This meant that their responding mechanisms were diverse, and were similar to the mechanisms of stress response of rice bZIP family genes [35]. More importantly, the response of the TGA members of Groups I, II, III, V and VII to SMV infection in the unifoliate leaves provided evidence of their novel function as a regulator of the virus stress process (Figure 2 and Figure S3). Besides, due to AtTGA1, 2, 4, 5 and 6 
were involved in the plant defense response in Arabidopsis [5], the closer the phylogenetic relationships between soybean TGA TFs were in Groups I and VII (Figure 1 and Figure S2), and they might also be required for self-defense in soybean or SMV successful colonization in a host. These findings provided a reference for the response of GmTGA genes in a virus-susceptible soybean in the face of infection by a single-stranded RNA virus.

\subsection{Potentials of GmTGA8 and GmTGA19 in Improving Soybean Resistance to SMV}

In the present study, against our expectations, most GmTGA genes from a hypersusceptible soybean inhibited SMV multiplication in inoculated leaves of $N$. benthamiana (Table 2). It indicated that GmTGA genes were not responsible for the susceptibility of hyper-susceptible soybean to SMV, but for the basal resistance to SMV, and they seemed to be functionally redundant for coping with SMV stress. In Arabidopsis, the knockout of Arabidopsis TGA2, TGA5 and TGA6, which belonged to the same group, revealed their redundant and essential roles in SAR [12]. While in soybean, the functional GmTGA proteins belonged to different groups in a phylogenetic relationship (Figure S2), suggesting the functional differentiation of the TGA paralogous genes in soybean was not obvious in response to an SMV attack. In contrast, tobacco bZIP TF TGA2.2 and TGA2.1 had distinct roles in the plant defense response and plant development, which indicated that tobacco TGA TFs possessed functional diversity [36]. In addition, overexpression of AtTGA2 conferred partial resistance to soybean cyst nematode in transgenic soybean hairy roots, AtTGA9 and AtTGA10 were involved in ROS-mediated responses to bacterial PAMP flg22 [21,37]. However, the reports on the functions of GmTGA TFs under pathogen attack or abiotic stress were very limited; for instance, GmTGA17 enhanced tolerance to drought and salt stress in both transgenic Arabidopsis plants and soybean hairy roots [38]. Thus, the function of GmTGA paralogous genes for growth development or resistance/tolerance to different stresses need to be further studied. In fact, we found that the GmTGA genes exhibited various degrees in inhibiting SMV replication (Table 2). Compared to the overexpression of the SMV coat protein, which increased the resistance to SMV in transgenic soybean [39], GmTGA8 and GmTGA19 performed better on inhibiting SMV multiplication than others. Therefore, TGA TFs possessed special roles in pathogen attack, and it was worthwhile to further study the function of GmTGA8 and GmTGA19 by transgene in soybean.

It was not difficult to find that GmTGA 8 was grouped with AtTGA1 and AtTGA4, and GmTGA19 was grouped with AtTGA2, AtTGA5 and AtTGA6 according to the phylogenetic relationship (Figure S2). As known to all, the Arabidopsis TGA TFs (TGA2, TGA5 and TGA6) play positive roles in the self-defense response, which interacted with NPR1 (NONEXPRESSOR OF PR GENES 1) to regulate the expression of $P R-1$ for gaining SAR [11,12]. Similarly, AtTGA1 and AtTGA4 also participated in plant immunity independent of NPR1 mediated SA-dependent defense response [13-15]. Although it was not clear whether the resistance mechanism of GmTGA8 and GmTGA19 in soybean was similar to that in Arabidopsis (Figure S1), the results of the predicted protein interaction network indirectly supported that NPR1 was the potential partner of GmTGA8 and GmTGA19 (Figure 4), and implied that GmTGA genes may also rely on the NPR1 pathway. Notably, WRKY8 and WRKY41 were the candidate partners of GmTGA19 rather than GmTGA8, and this WRKYTGA pair was also involved in activating the expression of SA-dependent defense genes in the charge of SAR in Arabidopsis [40,41]. The mechanism of GmTGA genes regulating the SMV disease process needs to be explored in the next step. Additionally, what the functions of GmTGA genes are in hyper-resistant soybean needs to be explored in the future.

\section{Materials and Methods}

\subsection{Plant Materials and SMV Strain}

SMV-susceptible soybean cultivar NN1138-2 was grown in an insect-free greenhouse with a $16 \mathrm{~h}$ photoperiod at $25^{\circ} \mathrm{C}$. SMV isolate $4278-1$ was provided by the National Center for Soybean Improvement (Nanjing Agricultural University, Nanjing, China). 


\subsection{Identification, Cloning and Sequence Analysis of GmTGA}

The amino acid sequences of Arabidopsis TGA TFs were retrieved from the Arabidopsis Information Resource (https://www.Arabidopsis.org, accessed on 17 June 2018). Two different approaches were used to mine the TGA TFs in soybean: (i) the BLASTP search (e-value $1 \times 10^{-20}$ ) was performed using Arabidopsis TGA TFs sequences; (ii) the Hidden Markov model (HMM) scan was performed using an HMM profile, which was built by the alignment of a set of Arabidopsis TGA TFs sequences. A final unique set of the protein sequences identified using the above approaches was further scanned for the presence of bZIP and DOG1 domains based on SMART [42] and Pfam [43] analysis. Only the sequences containing these domains were retained. For cloning the TGA TFs from soybean cultivar NN1138-2, the total RNA of the leaf, stem, root hairs and seed were separately extracted using the RNA isolation kit (Tiangen, Beijing, China) and the RNA mixture served as the template for reverse transcription using the PrimeScript ${ }^{\mathrm{TM}}$ II 1st strand cDNA synthesis kit (Takara, Dalian, China). The PCR reaction was performed using Phanta Max Super-Fidelity DNA polymerase (Vazyme, Nanjing, China) according to the recommended procedure. Another alternative amplification method was adopted while the PCR products could not be obtained from cDNA, that is, the DNA instead of the cDNA served as the template of the PCR reaction. The DNA was extracted using the DNAsecure plant kit (Tiangen, Beijing, China). The above primers used were listed in Table S1. All PCR products were purified using a gel extraction kit (Omega, Guangzhou, China) and were further ligated to the $\mathrm{T}$ vector using the pClone007 blunt vector kit (Tsingke, Nanjing, China) for sequencing (General Biosystems, Chuzhou, China). The coding sequences of all TGA TFs derived from NN1138-2 were predicted through comparing to the corresponding sequences of TGA TFs in Williams 82. The molecular weights and theoretical isoelectric points of TGA proteins were predicted by the ExPASy server [44].

\subsection{Phylogenetic Analysis}

Multiple alignments of the GmTGA amino acid sequences were performed using ClustalW with the default options in MEGA Version 6.0 [45,46]. Phylogenetic trees were constructed based on the neighbor-joining (NJ) method with the p-distance model and maximum likelihood analysis with a bootstrap of 1000 replicates.

\subsection{SMV Inoculation and Sample Collection}

SMV isolate 4278-1 was increased and maintained on a highly susceptible soybean NN1138-2. Young symptomatic leaves were homogenized in $0.01 \mathrm{M}$ phosphate buffer (PH 7.4) using a sterilized mortar with pestle and the tissue homogenates were filtered with two layers of cheesecloth. The fully developed unifoliate leaves of the soybean cultivar NN1138-2 were further mechanically inoculated by the above-mentioned filtered sap mixed with a small amount of carborundum powder (600-mesh) and the mock $(0.01 \mathrm{M}$ phosphate buffer, PH 7.4) inoculation as control. The inoculated leaves were collected at $0,1,2,4,8,12$, 24 and $72 \mathrm{~h}$ post-inoculation (hpi), respectively. Meanwhile, the fully developed unifoliate leaves of some untreated soybean plants were also collected. All the samples were stored at $-80{ }^{\circ} \mathrm{C}$ immediately after freezing in liquid nitrogen.

\subsection{The Quantitative Real-Time PCR Analysis}

The total RNA was extracted from the untreated and treated unifoliate leaves of soybean cultivar NN1138-2 using an RNA isolation kit (Tiangen, Beijing, China). The corresponding first-strand cDNA was synthesized (PrimeScript ${ }^{\mathrm{TM}} \mathrm{RT}$ reagent Kit, TAKARA, Dalian, China) and used for the quantitative real-time polymerase chain reaction (qRTPCR). The qRT-PCR was performed using a LightCycler 480II(ROCHE) in conjunction with the SYBR ${ }^{\circledR}$ Premix Ex Taq ${ }^{\mathrm{TM}}$ II (Tli RNaseH Plus, TAKARA, Dalian, China). Each reaction consisted of $5 \mu \mathrm{L}$ of SYBR ${ }^{\circledR}$ Premix Ex Taq ${ }^{\text {TM }}$ II, $0.2 \mu \mathrm{L}$ of each $10 \mu \mathrm{M}$ gene-specific forward primer and reverse primer, $2 \mu \mathrm{L}$ cDNA in a final volume of $10 \mu \mathrm{L}$. The primer pairs used for qRT-PCR are shown in Table S2 and the primers of the reference genes GmEF1B 
and GmActin 11 are taken from the literature [47]. The protocol was as follows: $30 \mathrm{~s}$ of initial denaturation at $95^{\circ} \mathrm{C}$; the samples were then subjected to cycling parameters of $95^{\circ} \mathrm{C}$ for $5 \mathrm{~s}$ (heating rate: $4.4{ }^{\circ} \mathrm{C} / \mathrm{s}$ ), $60^{\circ} \mathrm{C}$ for $30 \mathrm{~s}$ (heating rate: $2.2^{\circ} \mathrm{C} / \mathrm{s}$ ) (for 40 cycles). The relative expression of the gene was calculated using the $2^{-\Delta \Delta \mathrm{Ct}}$ method [48]. Three technical replicates in each of the three biological replicates for each sample were taken for qRT-PCR analysis.

\subsection{Construction of Transient Overexpression Constructs}

The vector pEarleygate103 was digested with restriction enzymes XhoI and PmlI, then the green fluorescent protein (GFP) gene and SMV coat protein $(c p)$ gene were cloned into the pEarleygate103 vector by the one-step recombination method, respectively (ClonExpress II One Step Cloning Kit, Vazyme, China), that is, p103-GFP (empty vector) and p103-CP (positive control). Moreover, the coding sequences of individual GmTGA genes were amplified from the above-mentioned $\mathrm{T}$ vectors ( $\mathrm{pClone} 007$ blunt vector), which contain all GmTGA genes sequences using their corresponding primers (Table S3), and were further cloned into the p103-GFP vector using the same restriction enzyme cutting site by the one-step recombination method, that is, the p103-GmTGA genes constructs.

\subsection{Transient Assay in N. benthamiana, SMV Inoculation and ELISA Detection}

For Agrobacterium-mediated transient overexpression of candidate genes in $N$. benthamiana, an individual overexpression construct was transformed into the Agrobacterium tumefaciens strain GV3101 through electroporation and the bacterial cultures were grown overnight $\left(28^{\circ} \mathrm{C}, 200 \mathrm{rpm}\right)$, pelleted by centrifugation and then resuspended in $10 \mathrm{mM} \mathrm{MgCl}_{2}$. The suspension was diluted to OD600 $=0.5$ with a solution containing a final concentration of $10 \mathrm{mM} \mathrm{MgCl}_{2}, 10 \mathrm{mM} \mathrm{MES} \mathrm{pH} \mathrm{5.7,} \mathrm{and} 150 \mu \mathrm{M}$ acetosyringone and was then infiltrated into 4-week-old leaves of $N$. benthamiana with a $1 \mathrm{~mL}$ needleless syringe. For SMV inoculation, the SMV isolate 4278-1-infected soybean leaves were homogenized in $0.01 \mathrm{M}$ phosphate buffer ( $\mathrm{PH} 7.4$ ) in a grinder and the tissue homogenates were filtered with two layers of cheesecloth. Then, Agrobacterium-infiltrated leaves of N. benthamiana were mechanically inoculated with the filtered sap. For ELISA assay, the SMV antiserum (polyclonal rabbit antibodies) of ELISA was purchased from ACD Inc. (cat \#V094-R1, Beijing, China) and the manufacturer's instructions were followed for the next steps.

\subsection{Conserved Motifs and NLS Analysis in GmTGA Protein}

Multiple expectation maximization for motif elicitation (MEME) was employed to identify and analyze the conserved motifs of the GmTGA sequences and only the motifs with E-values $<0.05$ as well as with no overlap with each other were reported. The NLS was predicted by the NLStradamus online tool [33].

\subsection{Protein-Protein Interaction (PPI) Network Analysis}

The search tool for the retrieval of the interacting genes (STRING) (https:/ / string-db. org, accessed on 1 March 2021) database was applied to predict functional interactions of proteins [49]. Active interaction sources, including databases, experiments, neighborhood, gene fusion, co-occurrence, textmining and co-expression as well as species limited to "Glycine max" and an interaction score $>0.4$ were applied to construct the PPI networks.

\subsection{Statistical Analysis}

The ELISA and qRT-PCR data were analyzed for their means, standard deviations, and single-factor analyses of variance using the SPSS software (version 18.0). Duncan's new multiple range tests were performed to determine any significant difference among various treatments using the significance level of $\alpha=0.05$. 


\section{Conclusions}

A total of 27 GmTGA genes isolated from an SMV hyper-susceptible soybean NN1138-2 were identified through homologous cloning. Expression analysis showed that most of them participated in response to SMV stress. Transient overexpression of SMV responding to GmTGA genes in N. benthamiana exhibited the basal resistance to SMV rather than promoting SMV-multiplication. It indicated that GmTGA genes were not responsible for the susceptibility of hyper-susceptible soybean to SMV, but for the basal resistance to SMV. For GmTGA8 and GmTGA19 especially, they possessed similar motifs distribution and a nuclear localization sequence. Moreover, according to protein-protein networks prediction analysis, NPR-class proteins were their main partners. Taken together, the unveiling of the roles of soybean TGA genes for inhibiting SMV multiplication provided new insights into improving SMV-resistance in susceptible soybeans.

Supplementary Materials: The following are available online at https:/ /www.mdpi.com/article/ 10.3390/ijms222111329/s1, Figure S1: Schematic representation of TGA TFs involved in pathogeninduced basal resistance, susceptibility and systemic acquired resistance (SAR). Figure S2: Phylogenetic analyses of TGA proteins from Arabidopsis and soybean. Figure S3: Expression profiles of GmTGA genes of soybean NN1138-2 before and after SMV inoculation at 1, 2, 4, 8, 12, 24 and $72 \mathrm{~h}$ post-inoculation (hpi). Table S1: The primers are used to amplify TGA TFs from soybean cultivar NN1138-2. Table S2: The qRT-PCR primers for the 27 GmTGA genes of soybean cultivar NN1138-2. Table S3: The construction primers of transient overexpression constructs.

Author Contributions: J.G. and H.J. conceived and designed the method and experiments; H.J., S.G. and K.L. performed the experiments, and H.J. analyzed the data; J.G. and H.J. drafted and revised the manuscript; J.G. contributed reagents/materials and interpretation of the results. All authors have read and agreed to the published version of the manuscript.

Funding: This work was financially supported through the grants from the Natural Science Foundation of China (31801388).

Institutional Review Board Statement: Not applicable.

Informed Consent Statement: Not applicable.

Data Availability Statement: All data are available upon reasonable request.

Acknowledgments: This work was supported by the Natural Science Foundation of China (31801388).

Conflicts of Interest: The authors declare no conflict of interest.

\section{Abbreviations \\ TF transcription factor \\ SMV Soybean mosaic virus \\ N. benthamiana Nicotiana benthamiana}

\section{References}

1. Chittem, K.; Yajima, W.R.; Goswami, R.S.; del Río Mendoza, L.E. Transcriptome analysis of the plant pathogen Sclerotinia sclerotiorum interaction with resistant and susceptible canola (Brassica napus) lines. PLoS ONE 2020, 15, e0229844. [CrossRef]

2. Zhang, L.; Shang, J.; Wang, W.; Du, J.; Li, K.; Wu, X.; Yu, L.; Liu, C.; Khaskheli, M.I.; Yang, W. Comparison of Transcriptome Differences in Soybean Response to Soybean Mosaic Virus under Normal Light and in the Shade. Viruses 2019, 11, 793. [CrossRef] [PubMed]

3. Singh, K.B.; Foley, R.C.; Oñate-Sánchez, L. Transcription factors in plant defense and stress responses. Curr. Opin. Plant Biol. 2002, 5, 430-436. [CrossRef]

4. de-Leon, S.B.-T.; Davidson, E.H. Gene regulation: Gene control network in development. Annu. Rev. Biophys. Biomol. Struct. 2007, 36, 191-212. [CrossRef] [PubMed]

5. Jakoby, M.; Weisshaar, B.; Dröge-Laser, W.; Vicente-Carbajosa, J.; Tiedemann, J.; Kroj, T.; Parcy, F. bZIP transcription factors in Arabidopsis. Trends Plant Sci. 2002, 7, 106-111. [CrossRef]

6. Katagiri, F.; Lam, E.; Chua, N.-H. Two tobacco DNA-binding proteins with homology to the nuclear factor CREB. Nature 1989, 340, 727-730. [CrossRef] [PubMed] 
7. Krawczyk, S.; Thurow, C.; Niggeweg, R.; Gatz, C. Analysis of the spacing between the two palindromes of activation sequence-1 with respect to binding to different TGA factors and transcriptional activation potential. Nucleic Acids Res. 2002, 30, 775-781. [CrossRef] [PubMed]

8. Xiang, C.; Miao, Z.; Lam, E. DNA-binding properties, genomic organization and expression pattern of TGA6, a new member of the TGA family of bZIP transcription factors in Arabidopsis thaliana. Plant Mol. Biol. 1997, 34, 403-415. [CrossRef]

9. Chern, M.; Bai, W.; Ruan, D.; Oh, T.; Chen, X.; Ronald, P.C. Interaction specificity and coexpression of rice NPR1 homologs 1 and 3 (NH1 and NH3), TGA transcription factors and Negative Regulator of Resistance (NRR) proteins. BMC Genom. 2014, 15, 1-20. [CrossRef]

10. Gatz, C. From pioneers to team players: TGA transcription factors provide a molecular link between different stress pathways. Mol. Plant-Microbe Interact. 2013, 26, 151-159. [CrossRef]

11. Zhang, Y.; Fan, W.; Kinkema, M.; Li, X.; Dong, X. Interaction of NPR1 with basic leucine zipper protein transcription factors that bind sequences required for salicylic acid induction of the PR-1 gene. Proc. Natl. Acad. Sci. USA 1999, 96, 6523-6528. [CrossRef]

12. Zhang, Y.; Tessaro, M.J.; Lassner, M.; Li, X. Knockout analysis of Arabidopsis transcription factors TGA2, TGA5, and TGA6 reveals their redundant and essential roles in systemic acquired resistance. Plant Cell 2003, 15, 2647-2653. [CrossRef]

13. Després, C.; Chubak, C.; Rochon, A.; Clark, R.; Bethune, T.; Desveaux, D.; Fobert, P.R. The Arabidopsis NPR1 disease resistance protein is a novel cofactor that confers redox regulation of DNA binding activity to the basic domain/leucine zipper transcription factor TGA1. Plant Cell 2003, 15, 2181-2191. [CrossRef]

14. Zhou, J.-M.; Trifa, Y.; Silva, H.; Pontier, D.; Lam, E.; Shah, J.; Klessig, D.F. NPR1 differentially interacts with members of the TGA/OBF family of transcription factors that bind an element of the PR-1 gene required for induction by salicylic acid. Mol. Plant-Microbe Interact. 2000, 13, 191-202. [CrossRef]

15. Shearer, H.L.; Cheng, Y.T.; Wang, L.; Liu, J.; Boyle, P.; Després, C.; Zhang, Y.; Li, X.; Fobert, P.R. Arabidopsis clade I TGA transcription factors regulate plant defenses in an NPR1-independent fashion. Mol. Plant-Microbe Interact. 2012, 25, 1459-1468. [CrossRef]

16. Sun, T.; Busta, L.; Zhang, Q.; Ding, P.; Jetter, R.; Zhang, Y. TGACG-BINDING FACTOR 1 (TGA 1) and TGA 4 regulate salicylic acid and pipecolic acid biosynthesis by modulating the expression of SYSTEMIC ACQUIRED RESISTANCE DEFICIENT 1 (SARD 1) and CALMODULIN-BINDING PROTEIN 60g (CBP 60g). New Phytol. 2018, 217, 344-354. [CrossRef]

17. Rahman, T.A.; Oirdi, M.E.; Gonzalez-Lamothe, R.; Bouarab, K. Necrotrophic pathogens use the salicylic acid signaling pathway to promote disease development in tomato. Mol. Plant Microbe Interact. 2012, 25, 1584-1593. [CrossRef]

18. Choi, J.; Huh, S.U.; Kojima, M.; Sakakibara, H.; Paek, K.-H.; Hwang, I. The cytokinin-activated transcription factor ARR2 promotes plant immunity via TGA3/NPR1-dependent salicylic acid signaling in Arabidopsis. Dev. Cell 2010, 19, 284-295. [CrossRef]

19. Kesarwani, M.; Yoo, J.; Dong, X. Genetic interactions of TGA transcription factors in the regulation of pathogenesis-related genes and disease resistance in Arabidopsis. Plant Physiol. 2007, 144, 336-346. [CrossRef]

20. Murmu, J.; Bush, M.J.; DeLong, C.; Li, S.; Xu, M.; Khan, M.; Malcolmson, C.; Fobert, P.R.; Zachgo, S.; Hepworth, S.R. Arabidopsis basic leucine-zipper transcription factors TGA9 and TGA10 interact with floral glutaredoxins ROXY1 and ROXY2 and are redundantly required for anther development. Plant Physiol. 2010, 154, 1492-1504. [CrossRef]

21. Noshi, M.; Mori, D.; Tanabe, N.; Maruta, T.; Shigeoka, S. Arabidopsis clade IV TGA transcription factors, TGA10 and TGA9, are involved in ROS-mediated responses to bacterial PAMP flg22. Plant Sci 2016, 252, 12-21. [CrossRef]

22. Chuang, C.-F.; Running, M.P.; Williams, R.W.; Meyerowitz, E.M. The PERIANTHIA gene encodes a bZIP protein involved in the determination of floral organ number in Arabidopsis thaliana. Genes Dev. 1999, 13, 334-344. [CrossRef]

23. Hill, J.H.; Whitham, S.A. Control of virus diseases in soybeans. Adv. Virus Res. 2014, 90, 355-390.

24. Li, K.; Zhi, H.J. Advances in resistance to Soybean mosaic virus disease in soybean. Soybean Sci. 2016, 35, 525-530.

25. Hill, J.H. Soybean mosaic virus. In Compendium of Soybean Diseases, 4th ed.; Hartman, G.L., Sinclair, J.B., Rupe, J.C., Eds.; APS Press: St. Paul, MN, USA, 1999; pp. 70-71.

26. Ross, J. Effect of Aphid-Transmitted Soybean Mosaic Virus on Yields of Closely Related Resistant and Susceptible Soybean Lines 1. Crop Sci. 1977, 17, 869-872. [CrossRef]

27. Hajimorad, M.R.; Domier, L.L.; Tolin, S.A.; Whitham, S.A.; Saghai Maroof, M.A. Soybean mosaic virus: A successful potyvirus with a wide distribution but restricted natural host range. Mol. Plant Pathol. 2018, 19, 1563-1579. [CrossRef]

28. Jiang, H.; Li, K.; Dou, D.; Gai, J. Characterization of a soybean mosaic virus variant causing different diseases in Glycine max and Nicotiana benthamiana. Arch. Virol. 2017, 162, 549-553. [CrossRef]

29. Almeida, Á.M.; Sakai, J.; Souto, E.R.; Kitajima, E.W.; Fukuji, T.S.; Hanada, K. Mosaic in Senna occidentalis in Southern Brazil induced by a new strain of Soybean mosaic virus. Fitopatol. Bras. 2002, 27, 151-156. [CrossRef]

30. Christie, S.R.; Crawford, W.E. Plant virus range of Nicotiana benthamiana. Plant Dis. Report. 1978, 62, $20-22$.

31. Schmutz, J.; Cannon, S.B.; Schlueter, J.; Ma, J.; Mitros, T.; Nelson, W.; Hyten, D.L.; Song, Q.; Thelen, J.J.; Cheng, J. Genome sequence of the palaeopolyploid soybean. Nature 2010, 463, 178. [CrossRef]

32. Ullah, I.; Magdy, M.; Wang, L.; Liu, M.; Li, X. Genome-wide identification and evolutionary analysis of TGA transcription factors in soybean. Sci. Rep. 2019, 9, 11186. [CrossRef] [PubMed]

33. Nguyen Ba, A.N.; Pogoutse, A.; Provart, N.; Moses, A.M. NLStradamus: A simple Hidden Markov Model for nuclear localization signal prediction. BMC Bioinform. 2009, 10, 202. [CrossRef] [PubMed] 
34. Pieterse, C.M.; Van Loon, L. NPR1: The spider in the web of induced resistance signaling pathways. Curr. Opin. Plant Biol. 2004, 7, 456-464. [CrossRef] [PubMed]

35. Nijhawan, A.; Jain, M.; Tyagi, A.K.; Khurana, J.P. Genomic survey and gene expression analysis of the basic leucine zipper transcription factor family in rice. Plant Physiol. 2008, 146, 333-350. [CrossRef]

36. Thurow, C.; Schiermeyer, A.; Krawczyk, S.; Butterbrodt, T.; Nickolov, K.; Gatz, C. Tobacco bZIP transcription factor TGA2.2 and related factor TGA2.1 have distinct roles in plant defense responses and plant development. Plant J. 2005, 44, 100-113. [CrossRef]

37. Matthews, B.F.; Beard, H.; Brewer, E.; Kabir, S.; Macdonald, M.H.; Youssef, R.M. Arabidopsis genes, AtNPR1, AtTGA2 and AtPR-5, confer partial resistance to soybean cyst nematode (Heterodera glycines) when overexpressed in transgenic soybean roots. BMC Plant Biol. 2014, 14, 96. [CrossRef]

38. Li, B.; Liu, Y.; Cui, X.-Y.; Fu, J.-D.; Zhou, Y.-B.; Zheng, W.-J.; Lan, J.-H.; Jin, L.-G.; Chen, M.; Ma, Y.-Z. Genome-Wide Characterization and Expression Analysis of Soybean TGA Transcription Factors Identified a Novel TGA Gene Involved in Drought and Salt Tolerance. Front. Plant Sci. 2019, 10, 549. [CrossRef]

39. Wang, X.; Eggenberger, A.L.; Fwjr, N.; Hill, H.J. Pathogen-derived transgenic resistance to soybean mosaic virus in soybean. Mol. Breed. 2001, 8, 119-127. [CrossRef]

40. Pieterse, C.M.; Zamioudis, C.; Berendsen, R.L.; Weller, D.M.; Van Wees, S.C.; Bakker, P.A. Induced systemic resistance by beneficial microbes. Annu. Rev. Phytopathol. 2014, 52, 347-375. [CrossRef]

41. Hussain, R.M.; Sheikh, A.H.; Haider, I.; Quareshy, M.; Linthorst, H.J. Arabidopsis WRKY50 and TGA transcription factors synergistically activate expression of PR1. Front. Plant Sci. 2018, 9, 930. [CrossRef]

42. Letunic, I.; Bork, P. 20 years of the SMART protein domain annotation resource. Nucleic Acids Res. 2017, 46, D493-D496. [CrossRef]

43. El-Gebali, S.; Mistry, J.; Bateman, A.; Eddy, S.R.; Luciani, A.; Potter, S.C.; Qureshi, M.; Richardson, L.J.; Salazar, G.A.; Smart, A. The Pfam protein families database in 2019. Nucleic Acids Res. 2018, 47, D427-D432. [CrossRef]

44. Gasteiger, E.; Gattiker, A.; Hoogland, C.; Ivanyi, I.; Appel, R.D.; Bairoch, A. ExPASy: The proteomics server for in-depth protein knowledge and analysis. Nucleic Acids Res. 2003, 31, 3784-3788. [CrossRef]

45. Thompson, J.D.; Higgins, D.G.; Gibson, T.J. CLUSTAL W: Improving the sensitivity of progressive multiple sequence alignment through sequence weighting, position-specific gap penalties and weight matrix choice. Nucleic Acids Res. 1994, 22, 4673-4680. [CrossRef]

46. Tamura, K.; Stecher, G.; Peterson, D.; Filipski, A.; Kumar, S. MEGA6: Molecular evolutionary genetics analysis version 6.0. Mol. Biol. Evol. 2013, 30, 2725-2729. [CrossRef]

47. Ma, S.; Niu, H.; Liu, C.; Zhang, J.; Hou, C.; Wang, D. Expression stabilities of candidate reference genes for RT-qPCR under different stress conditions in soybean. PLoS ONE 2013, 8, e75271. [CrossRef]

48. Livak, K.J.; Schmittgen, T.D. Analysis of relative gene expression data using real-time quantitative PCR and the $2^{-\Delta \Delta C T}$ method. Methods 2001, 25, 402-408. [CrossRef]

49. Szklarczyk, D.; Gable, A.L.; Lyon, D.; Junge, A.; Wyder, S.; Huerta-Cepas, J.; Simonovic, M.; Doncheva, N.T.; Morris, J.H.; Bork, P. STRING v11: Protein-protein association networks with increased coverage, supporting functional discovery in genome-wide experimental datasets. Nucleic Acids Res. 2019, 47, D607-D613. [CrossRef] 